\title{
Pérdida de visión
}

\author{
María Lafarga Travera , Antonio Navarro Ballestera, Susana de Lázaro de Molina ${ }^{a}$, \\ José Hermenegildo García-Vila
}

\begin{abstract}
a Servicio de Radiología Diagnóstica y Terapéutica. Hospital General de Castellón. Castellón (España).

b Jefe de Servicio de Radiología Diagnóstica y Terapéutica. Hospital General de Castellón. Castellón (España).

Correspondencia: María Lafarga Traver. Correo electrónico: marialafargatraver@gmail.com

Recibido el 15 de noviembre de 2012.

Aceptado para su publicación el 27 de junio de 2013.
\end{abstract}

\section{RESUMEN}

Paciente varón de 81 años que acude a urgencias refiriendo pérdida de visión en el campo visual superior izquierdo desde hace unos días. A la exploración oftalmológica, se evidenciaba ausencia de percepción de luz en el campo superior del ojo izquierdo. En el fondo de ojo aparecía una masa retiniana con origen a nivel nasal inferior. Se realiza una ecografía, donde se identifica una lesión ocupante de espacio (melanoma coroideo) con desprendimiento de retina asociado.

Palabras clave: Neoplasias de la coroides. Desprendimiento de retina. Baja visión.

\begin{abstract}
Loss of sight

81 years old male patient who went to the emergency department after beginning to suffer loss of sight in the upper left visual field two days earlier. On ophthalmic examination, absence of light perception was evident in the upper left eye field. At the back of the eye there was a retinal mass originating at lower nasal level. An ultra sound was performed and a space occupying lesion (choroidal melanoma) with associated retinal detachment was identified.
\end{abstract}

Key words: Choroidal neoplasms. Retinal detachment. Low vision.

\section{INTRODUCCIÓN}

En un paciente con pérdida súbita de visión es recomendable realizar una valoración del grado de visión de cada ojo (escala de optotipos, cuenta de dedos...), reflejo fotomotor, motilidad ocular extrínseca y campo visual por confrontación; se deben identificar las modificaciones del aspecto externo del ojo (enrojecimiento, edema...); a continuación, realizar una oftalmoscopia directa para valorar disco óptico, vasos retinianos y mácula. Además de la exploración oftalmológica básica descrita anteriormente, podemos completar el estudio con el examen de fondo de ojo ${ }^{1,2}$.

En el caso de ver turbidez del vítreo, dificultad para visualizar el nervio óptico y una fina capa elevada de tejido retiniano, con pliegues, hay que sospechar un desprendimiento de retina ${ }^{3}$. En ocasiones, también podemos identificar una lesión dependiente de capas profundas del globo ocular. En estos casos, se puede ampliar el estudio con ecografía ocular y otras modalidades de imagen como Tomografia Computarizada (TC) y Resonancia Magnética (RM).

La ecografía ocular es de gran importancia en Atención Primaria. La realización es rápida, el coste mínimo y posee una sensibilidad diagnóstica elevada. Es el método primario de diagnóstico en el melanoma coroideo, aportando datos valiosos sobre la vascularización y tamaño del mismo, ya que el pronóstico y tratamiento dependen de este último ${ }^{4}$. El diagnóstico diferencial debe incluir: metástasis de pulmón y mama (tumores intraoculares más frecuentes), hemangiomas, nevus, hiperplasia de epitelio pigmentario, degeneración macular asociada a la edad (DMAE) o luxación del cristalino.

El melanoma coroideo es el tumor intraocular primario más frecuente en 
el adulto $^{5}$. La úvea (coroides, cuerpo ciliar e iris) constituye la túnica más vascularizada del globo ocular, lo cual facilita el asentamiento tumoral. Factores relacionados con un mayor riesgo de desarrollar un melanoma ocular son: melanocitosis (ocular o cutánea), síndrome de nevus displásico, pigmentación clara del iris, antecedentes personales o familiares de melanoma, nevus en piel o nevus preexistente de coroides ${ }^{6}$.

\section{OBSERVACIONES CLÍNICAS}

El caso que aportamos es el de un paciente varón de 82 años, sin alergias medicamentosas conocidas. No refiere hipertensión arterial, dislipemia ni diabetes. Diagnosticado de fibrilacion auricular paroxítica hace 3 años. Ictus en 2009 con defecto visual temporal derecho como secuela persistente. Hipertrofia benigna de próstata en tratamiento. No EPOC conocida ni intervenciones quirúrgicas. Su tratamiento habitual: Bisoprolol, Triptorelina. No fumador ni otros hábitos tóxicos. Barthel de 100.

Acude a urgencias por pérdida de visión en ojo izquierdo (Ol) sin otra clínica acompañante. En la exploración oftalmológica se evidencia reflejo pupilar y fotomotor directo consensuados y simétricos. Estudio de motilidad ocular extrínseca

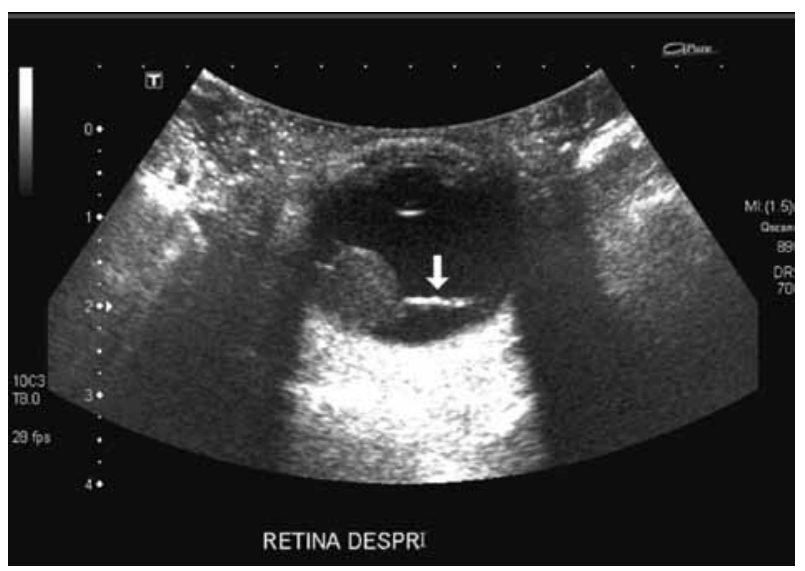

Figura 1. Ecografía ocular izquierda. Línea hiperecogénica, que flota libremente en humor vítreo posterior, en relación con desprendimiento de retina, ya conocido (flecha). Masa intraocular, redondeada / bilobulada, que protruye sobre humor vítreo, ubicada entre la lámina de retina desprendida y coroidesesclera en vertiente posteronasal del globo ocular. Ecos puntiformes internos en humor vítreo. dentro de la normalidad. Ausencia de percepción de luz en el campo visual temporal derecho (residual) ni a la iluminación superior de ojo izquierdo (de nueva aparición). Agudeza visual: Ojo Derecho (OD): 1; Ol:1/2. Biomicroscopia: conjuntiva normal, córnea clara, pupilas isocóricas y normorreactivas. No Tyndall. Fluoroscopia negativa. Presión intraocular (PIO) $16 \mathrm{mmHg}$. Se dilata la pupila con tropicamida 1\% para examen de fondo ocular, observando: masa retiniana con origen a nivel nasal anterior que cubre parcialmente la zona macular inferior, compatible con el defecto visual que el paciente relata. Se solicita ecografía ocular complementaria (figuras 1 y 2):

OI: Línea hiperecogénica, que flota libremente en humor vítreo posterior, en relación con desprendimiento de retina, ya conocido. Masa intraocular, redondeada / bilobulada, que protruye sobre humor vítreo, ubicada entre la lámina de retina desprendida y coroides-esclera en vertiente posteronasal del globo ocular. Mide $1 \mathrm{~cm} \times 8 \mathrm{~mm}$, está bien delimitada, isoecoica con respecto a la musculatura, sólida, vascularizada (el estudio Doppler muestra ondas espectrales arteriales intralesionales). Ecos puntiformes internos en humor vítreo. OD: sin alteraciones.

Tras el diagnóstico, el paciente fue remitido a su centro oncológico de referencia para tratamiento

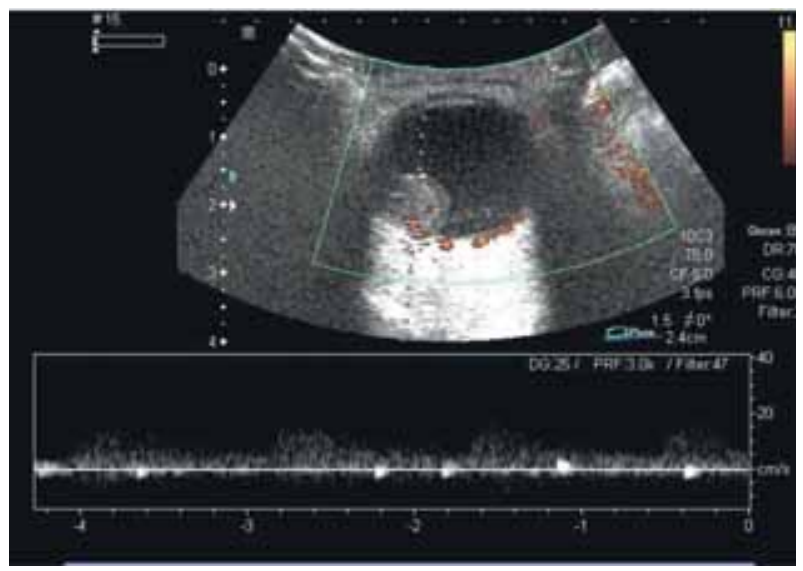

Figura 2. Ecografía doppler ocular izquierda. Vascularización de la masa intraocular, con ondas espectrales arteriales intralesionales. 
con braquiterapia local. Sin embargo, el paciente presentó un episodio de glaucoma agudo secundario al melanoma uveal (PIO: $48 \mathrm{mmHg}$ ) que requirió una enucleación ocular urgente del ojo izquierdo.

\section{COMENTARIOS}

En estadios iniciales el melanoma coroideo se presenta como una lesión aplanada o discretamente sobreelevada en la superficie uveal. Tiene una gran tendencia al crecimiento, observándose en fases avanzadas una morfología redondeada 0 "en champiñón" al romper la membrana de Bruch, asociando un desprendimiento de retina la mayoría de las veces.

El pronóstico dependerá fundamentalmente del tamaño tumoral: pequeño $(<10 \mathrm{~mm})$ y grande $(>10$ $\mathrm{mm})$. Otros factores a tener en cuenta serán la invasión del nervio óptico, el aumento de la PIO o las metástasis a distancia ${ }^{7}$.

En cuanto al tratamiento, deberá ser lo más conservador posible, dependiendo del tamaño, siendo de elección la braquiterapia con radioisótopos en menores de $10 \mathrm{~mm}$. De poseer un diámetro mayor, lo indicada es realizar una enucleación.

La detección temprana por parte del médico de familia, ya sea por oftalmoscopia o por pruebas complementarias, es fundamental para mejorar el pronóstico de los pacientes con pérdida de visión secundaria, entre otros al melanoma coroideo.

\section{BIBLIOGRAFÍA}

1. Sánchez JL, Díez C. Pérdida Brusca de Visión. Medynet [2003]. En:

http://www.medynet.com/usuarios/jraguilar/Manual\%20 de\%20urgencias\%20y\%20Emergencias/vision.pdf.

Consultado: 11/11/2012.

2. Seijas MC, Arnisen A. Pérdida de visión brusca. JANO. 2003; 64 (1469): 60-1.

3. Byer NE. Natural history of posterior vitreous detachment with early management as the premier line of defense against retinal detachment. Ophthalmology. 1994; 101: 1503-14.

4. Houle V, Bélair M, Allaire GS. AIRP Best Cases in Radiologic-Pathologic Correlation: Choroidal Melanoma. RadioGraphics. 2011; 31: 1231-6.

5. Tong KA, Osborn AG, Mamalis N, Harrie RP, Call NB. Ocular melanoma. AJNR. 1993; 14: 1359-66.

6. Mafee MF, Peyman GA, Grisolano JE, Fletcher ME, Spigos DG, Wehrli FW et al. Malignant uveal melanoma and simulating lesions: MR imaging evaluation. Radiology. 1986; 160: 773-80.

7. Cortez R, Totah AB, Pifano IA, Ramírez G. Melanoma Maligno Uveal Posterior. Clínica, Diagnostico y Tratamiento. Conceptos Actualizados. Rev Oftalmol Venez. 2003; 59 (4): 67-78. 